\title{
Predictors of 6-month health utility outcomes in survivors of acute respiratory distress syndrome
}

\author{
Samuel M Brown, ${ }^{1,2,3}$ Emily Wilson, ${ }^{1,2}$ Angela P Presson, ${ }^{4}$ Chong Zhang, ${ }^{4}$ \\ Victor D Dinglas, ${ }^{5,6}$ Tom Greene, ${ }^{4}$ Ramona 0 Hopkins, ${ }^{1,2,7}$ Dale M Needham, ${ }^{5,6,8}$ \\ with the National Institutes of Health NHLBI ARDS Network
}

- Additional material is published online only. To view please visit the journal online (http://dx.doi.org/10.1136/ thoraxjnl-2016-208560).

For numbered affiliations see end of article.

\section{Correspondence to} Dr Samuel M Brown, Shock Trauma Intensive Care Unit, 5121 South Cottonwood Street, Murray UT 84107, USA; Samuel.Brown@imail.org

Received 29 February 2016 Revised 3 June 2016

Accepted 18 June 2016 Published Online First 20 July 2016

\section{SLinked}

http://dx.doi.org/10.1136/ thoraxjnl-2016-209082

CrossMark

To cite: Brown SM, Wilson $\mathrm{E}$ Presson AP, et al.

Thorax 2017:72:311-317.

\section{ABSTRACT}

Background With improving short-term mortality in acute respiratory distress syndrome (ARDS),

understanding and improving quality of life (QOL) outcomes in ARDS survivors is a clinical and research priority. We sought to identify variables associated with $\mathrm{QOL}$, as measured by the EQ-5D health utility score, after ARDS using contemporary data science methods. Methods Analysis of prospectively acquired baseline variables and 6-month EQ-5D health utility scores for adults with ARDS enrolled in the ARDS Network LongTerm Outcomes Study (ALTOS). Penalised regression identified predictors of health utility, with results validated using 10 -fold cross-validation.

Results Among 616 ARDS survivors, several predictors were associated with 6-month EQ-5D utility scores, including two lifestyle factors. Specifically, older age, female sex, Hispanic/Latino ethnicity, current smoking and higher body mass index were associated with lower EQ-5D utilities, while living at home without assistance at baseline and AIDS were associated with higher EQ-5D utilities in ARDS survivors. No acute illness variables were associated with EQ-5D utility.

Conclusions Acute illness variables do not appear to be associated with postdischarge QOL among ARDS survivors. Functional independence and lifestyle factors, such as obesity and tobacco smoking, were associated with worse QOL. Future analyses of postdischarge health utility among ARDS survivors should incorporate measures of demographics and functional independence at baseline.

Trial registration numbers NCT00719446 (ALTOS), NCT00434993 (ALTA), NCT00609180 (EDEN/OMEGA), and NCT00883948 (EDEN); Post-results.

\section{INTRODUCTION}

Recent advances have substantially reduced shortterm mortality after acute respiratory distress syndrome (ARDS) ${ }^{1}$ leading to a greater focus on understanding and improving survivors' long-term physical, cognitive and mental health morbidities, and associated impairments in health-related quality of life (QOL). ${ }^{2}$

Multiple factors could potentially affect the recovery of ARDS survivors, including patient demographics, comorbidities, health habits, severity of the acute illness and intensive care unit (ICU) treatments. Understanding the association of such factors with patient outcomes is necessary to

\section{Key messages}

What is the key question?

- What variables available during hospitalisation are associated with 6-month health utility among acute respiratory distress syndrome survivors?

What is the bottom line?

- Prior degree of residential independence, and smoking and obesity are associated with 6-month healthy utility; acute illness severity is not.

\section{Why read on?}

- To understand potential predictors of health utility outcomes among acute respiratory distress syndrome survivors.

develop tailored interventions to improve ARDS patients' outcomes. ${ }^{3}$ Health utility scores represent an important summary measure of QOL; utility scores rate a given health state as a percentage of perfect health, where a score of 1 is perfect health and a score of 0 is considered equivalent to death, with exact scores for various possible health states in between assessed on the basis of large surveys of community-dwelling individuals.

Existing studies evaluating postdischarge QOL in ICU survivors have often included small sample sizes, used a single-centre design, evaluated only a relatively small number of possible predictors, and failed to use modern data science methods for evaluating associations. ${ }^{4-6}$ We therefore sought to evaluate a large, extensive dataset of potential predictors of health utility, measured at 6 months after ARDS, analysed with modern data science methods, using data from several multicentre, national ARDS Network clinical trials and the associated ARDS Network Long-Term Outcomes Study (ALTOS).

\section{METHODS}

Study design

We performed secondary analyses of data from ALTOS, ${ }^{7}$ which prospectively collected QOL outcomes for ARDS survivors from three ARDS Network multicenter, national randomised trials: 
albuterol for the treatment of ALI (ALTA; nebulised albuterol vs placebo), early versus delayed enteral nutrition (EDEN; early vs delayed feeding) and 'OMEGA' (nutritional supplements vs placebo). These three studies had similar enrolment criteria (see online supplementary data), including an identical prospective case-finding definition of ARDS. Relevant to our analysis, clinical trial eligibility criteria excluded patients with extreme obesity (body mass/height $>1 \mathrm{~kg} / \mathrm{cm}$ ), end-stage liver disease and end-stage AIDS on the grounds that their outcomes would be driven by factors other than ARDS. ALTOS enrolled ARDS survivors from 41 of 43 ARDS Network hospitals. The ARDS Network trials, ALTOS, and the present study were approved by the relevant institutional review boards, with informed consent obtained for patient participation in the ARDS Network trials and the ALTOS follow-up study.

\section{Primary outcome}

For this analysis, the primary outcome was the utility score from the EQ-5D-3L QOL instrument ${ }^{8}$ administered 6 months after ARDS. The EQ-5D-3L is a validated, reliable, widely used ${ }^{6} 9$ and recommended ${ }^{10}$ self-reported QOL instrument that evaluates whether respondents have no, mild, or serious problems in each of five domains: mobility, self-care, usual activities, pain/ discomfort and anxiety/depression. Responses are used to calculate an overall utility score based on US tariffs using a time-trade-off method whereby respondents indicate how much decrease in life span they would hypothetically accept to return from a given health state to perfect health. ${ }^{11}$ The resulting health utility score ranges from -0.11 (a state 'worse than death'; all domains with 'extreme' problems) to 1.00 (perfect health; all domains with 'no problems'). By way of illustration, a patient with 'moderate' pain or discomfort, but 'no problems' in any other domain would have a health utility of 0.83 ; a patient with 'some problems' in all domains would have a health utility of 0.60 and a patient with 'extreme' anxiety or depression and 'some problems' in all other domains would have a health utility of 0.38 . While a minimal important difference (MID) in EQ-5D is not established in ICU survivors, a difference of 0.050.09 is established for general populations. ${ }^{12} 13$

Because prior work suggests that proxy responses differ from ARDS survivor responses, ${ }^{14}$ we only used EQ-5D results obtained directly from patients in this analysis.

\section{Clinical predictors and covariates}

We evaluated 144 baseline predictor variables from the ARDS Network clinical trials and ALTOS (see online supplementary eTables 1-2 for complete list). These predictors included demographic variables, degree of independent living prior to hospital admission, comorbidities, alcohol ${ }^{15}$ and tobacco use history, APACHE III severity of illness score on the day before enrolment, Glasgow Coma Scale score on the first study day, available ICU-related treatment factors (including receipt of corticosteroids on the first study day), and daily fluid balance and clinical laboratory results. The degree of independent living was defined on an ordinal scale with scores of (1) home independently, to (2) home with informal help, to (3) home with professional help or living in an institution (eg, rehabilitation facility, skilled nursing facility or acute care hospital). For arterial blood gas values, we used all measures obtained on the day leading up to enrolment, including lowest, highest, mean and range (highest minus lowest) for each of $\mathrm{PaO}_{2}: \mathrm{FiO}_{2}$ ratio, $\mathrm{PaO}_{2}, \mathrm{pH}$ and $\mathrm{PaCO}_{2}$. Where potentially clinically relevant, the modelling of variables included use of quadratic terms (eg, for body mass index (BMI), $\mathrm{pH}$ and $\mathrm{PaCO}_{2}$ ) and statistical interaction terms (eg, minute ventilation with $\mathrm{PaCO}_{2}$ and serum creatinine with receipt of dialysis). For our primary analysis, we used only predictor variables available on day 1 or earlier, and we restricted the analysis to patients who survived with a 6-month follow-up assessment using EQ-5D. Further details related to modelling of predictor variables are included in the online supplementary data.

As an a priori sensitivity analysis, the primary analysis was repeated to include patients who died before 6-month follow-up, assigning them an EQ-5D utility score of 0 as done in prior research. ${ }^{16}$ Further details of this sensitivity analysis are available in the online supplementary data.

For a secondary analysis intended to evaluate predictors available at the time of hospital discharge, we studied only patients who were alive at hospital discharge. For this secondary analysis, we incorporated data available after day 1, including day 7 Brussels organ dysfunction scores, and ICU and hospital length of stay. Repeated measures of predictors, such as Brussels organ dysfunction measures, vital signs and ventilator settings from the first three study days were summarised using a variety of approaches (eg, mean, maximum and range) as outlined in the online supplementary data. We also performed a sensitivity analysis of this secondary analysis, repeating the secondary analysis to include patients who died before 6-month follow-up, assigning them an EQ-5D utility score of 0 as done in prior research. $^{16}$

\section{Statistical methods}

To select the predictor variables jointly associated with 6-month EQ-5D utility scores, we applied group least absolute shrinkage and selection operator (LASSO) penalised linear regression ${ }^{17}$ on all 144 candidate variables. Further details of the regression technique, including an analysis of the role of hospital site, are described in the online supplementary data. Because EQ-5D utilities are known to be negatively skewed, we applied the Box-Cox transformation to EQ-5D utilities and standardised them to have mean 0 and an SD of 1 so that interpretations could be made in SD increments. In order to validate the regression model results, we used 10 -fold cross-validation to estimate the root mean squared error (RMSE) and $\mathrm{R}^{2} \cdot{ }^{18}$ Variables selected from penalised regression were collectively fit using ordinary least squares linear regression to estimate CIs and $\mathrm{p}$ values, conditional on the variables selected, as explained further in the online supplementary data. Because penalised regression may select only one of several highly correlated variables, we also explored patterns of collinearity. Specifically, we evaluated correlation between selected predictors and unselected predictors. We also added key severity of illness covariates (admission APACHE III score, admission Brussels organ failure score, $\mathrm{PaO}_{2}: \mathrm{FiO}_{2}$ ratio and positive end expiratory pressure (PEEP)) to the primary model to see whether inclusion of those acute illness severity variables changed the model substantially (further details in online supplementary data).

While we acknowledge that retrospective reports of baseline EQ-5D are potentially unreliable, ${ }^{19}$ in an exploratory, post hoc analysis, we evaluated an a-priori subset of 195 patients who had 'baseline' EQ-5D measured retrospectively, evaluating the effect of inclusion of baseline EQ-5D on the primary model (further details in online supplementary data).

We also compared the observed regression coefficients to the MID for EQ-5D. Because we used a Box-Cox transformation and standardised results, the translation of coefficients into the MID is inexact. However, from the median value of EQ-5D, a 
coefficient of 0.27 was associated with a change of EQ-5D of 0.05; we used that threshold to evaluate whether regression coefficients were compatible with at least minimal clinical importance.

As recommended in prior literature, ${ }^{20}$ we imputed missing values using single imputation for variables where missingness was $\leq 15 \%$ and excluded variables with missingness $>15 \%$ (see details of imputation strategy in online supplementary data).

We performed all analyses in the R Statistical Package, V.3.2.1 (http://www.r-project.org). Statistical significance was defined as $\mathrm{p}<0.05$.

\section{RESULTS}

As depicted in figure 1, a total of 1176 eligible patients were enrolled in the ARDS Network clinical trials, with 247 (21\%) dying before hospital discharge, 44 (4\%) dying after hospital discharge but before ALTOS reconsent, 31 (3\%) declining to participate and 156 (13\%) meeting ALTOS exclusion criteria (see online supplementary appendix 4). Among 698 eligible and consenting patients, $42(6 \%)$ died prior to 6-month follow-up, with 645 (98\%) of the 656 survivors having 6-month outcome assessments, and 616 (94\%) of 656 having complete EQ-5D results available (29 patients completed partial follow-up without fully completing EQ-5D). For the sensitivity analysis that included deceased patients, a total of 949 patients were available for analysis. Table 1 displays the distribution of baseline and outcome characteristics among study patients.

For the prespecified primary analysis of 6-month EQ-5D utility scores in ARDS survivors, nine variables remained in the final regression model (table 2), with seven having statistically significant associations (the other two variables, pulmonary comorbidity and nadir respiratory rate, demonstrated low coefficients and non-significant $\mathrm{p}$ values). Hospital site was not retained in the model. The selected primary model $\mathrm{R}^{2}$ in the full dataset was 0.15 and met the regression assumptions of linearity and homoscedasticity. ${ }^{21}$ The cross-validated RMSE was 0.975 , and the cross-validated $\mathrm{R}^{2}$ was 0.03 .

All seven predictors with $\mathrm{p}<0.05$ were baseline attributes; none were factors associated with severity of acute illness or ICU treatments, including mechanical ventilation parameters (eg, $\mathrm{PaO}_{2}: \mathrm{FiO}_{2}$ ratio or driving pressure). At the time of hospital admission for ARDS, residing at home but with informal caregiver help $(p<0.001)$ or requiring professional help (including residence at a skilled nursing facility or similar) $(\mathrm{p}=0.03)$ was associated with lower 6-month health utility versus residing at home independently. Older age, female sex, Hispanic/Latino ethnicity and higher baseline BMI were all associated with lower health utility at 6-month follow-up. Latino ethnicity varied by hospital, from $0 \%$ to $44 \%$.

Based on standard regression coefficients, the magnitude of associations of female sex, Latino ethnicity, baseline independence, AIDS and current smoking with the 6-month EQ-5D utility score were all greater than the estimated MID.

The 10 survivors with AIDS at hospital admission had higher health utility $(p=0.01)$ than patients without AIDS, but, notably, AIDS comorbidity $(\mathrm{N}=24)$ was associated with higher inpatient mortality $(33 \%$ vs $58 \%, \mathrm{p}=0.02)$. Being a current smoker (vs non-smoker) at the time of hospital admission was associated with lower 6-month EQ-5D utility scores $(\mathrm{p}<0.001)$ and lower mortality $(27 \%$ vs $39 \%, \mathrm{p}<0.001)$.

In evaluating potential collinearity (details in online supplementary data) ever smoking was moderately correlated with
Figure 1 Identification of subjects for present study. ARDS, acute respiratory distress syndrome; ALTA, albuterol for the treatment of ALI; ALTOS, ARDS Network Long-Term Outcomes Study; EDEN, early versus delayed enteral nutrition; SAILS, statins for acutely injured lungs from sepsis.

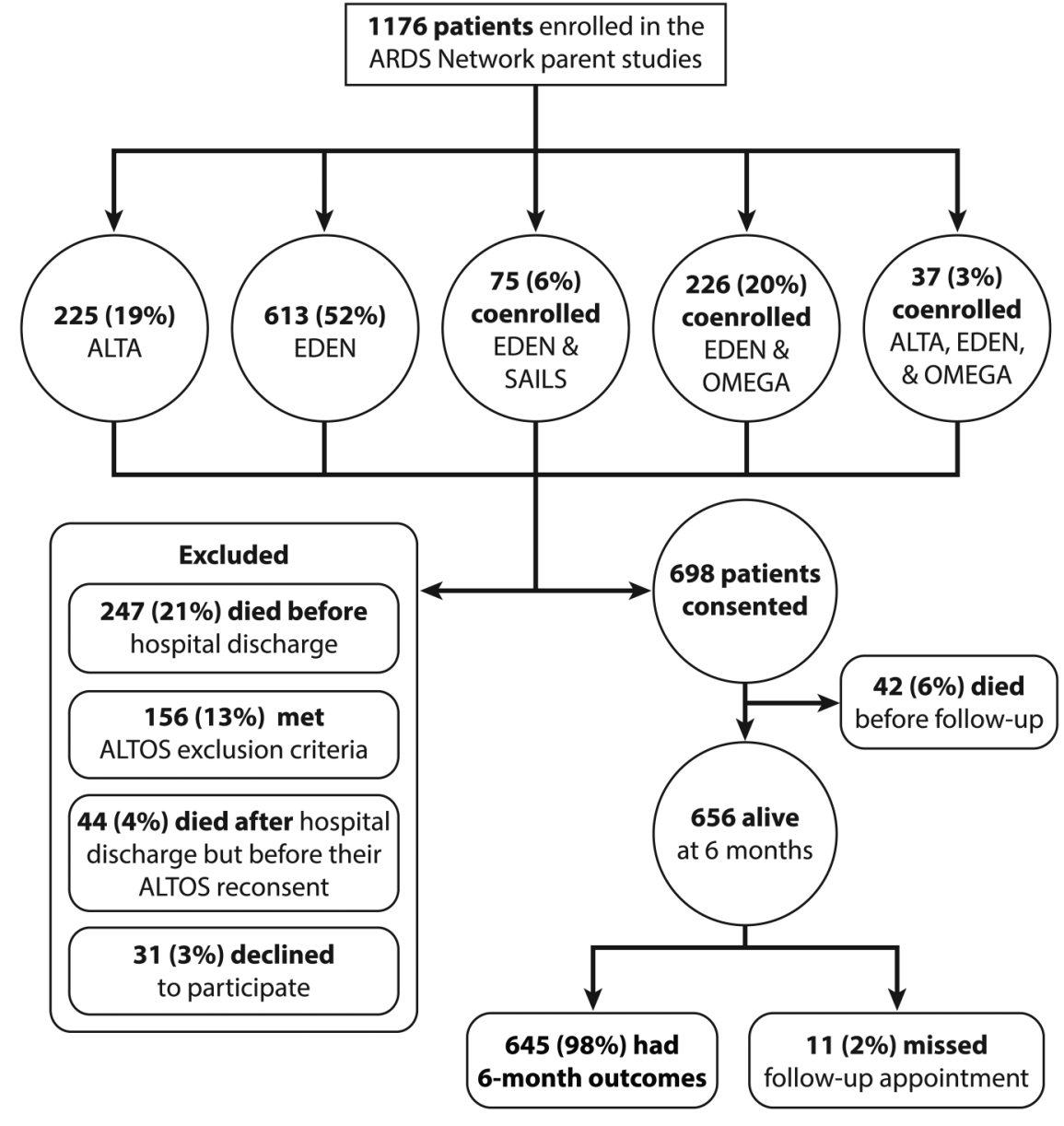

1176 patients enrolled in the

ARDS Network parent studies 
Table 1 Characteristics of Acute Respiratory Distress Syndrome (ARDS) survivors included in primary analysis

Values $(\mathrm{N}=645)$

Baseline demographic and clinical variables

Age (years); mean \pm SD $\quad 49 \pm 14$

Female sex; $\mathrm{n}(\%)$

$331(51 \%)$

Hispanic/Latino; $\mathrm{n}(\%)$

$56(9 \%)$

APACHE III; mean \pm SD

$85 \pm 26$

Race; $\mathrm{n}(\%)$

American Indian or Alaskan Native $\quad 5(1 \%)$

Asian $8(1 \%)$

White $507(77 \%)$

Black or African American 104 (16\%)

Native Hawaiian or other Pacific Islander $\quad 1(<1 \%)$

Not reported $17(3 \%)$

Lung injury category; $\mathrm{n}(\%)$

Trauma

$32(5 \%)$

Sepsis

Multiple transfusion

$106(16 \%)$

$15(2 \%)$

$72(11 \%)$

$387(60 \%)$

Pneumonia

$38(6 \%)$

Comorbidities; $\mathrm{n}(\%)$

Chronic dialysis

Leukaemia (AML, CML, ALL, multiple myeloma)

$14(2 \%)$

$11(2 \%)$

$5(1 \%)$

Non-Hodgkin's lymphoma

$4(1 \%)$

$49(8 \%)$

$3(<1 \%)$

$24(3 \%)$

$154(24 \%)$

$269(42 \%)$

$31(5 \%)$

$32(5 \%)$

$25(4 \%)$

$10(2 \%)$

$3(<1 \%)$

$73(11 \%)$

$46(7 \%)$

$18(3 \%)$

Peptic ulcer disease

Variables in the primary analysis

Residence prior to hospitalisation; $\mathrm{n}(\%)$

Home independently

$589(91 \%)$

$36(6 \%)$

$20(3 \%)$

Requiring professional help at home OR residence at

healthcare facility

AIDS; $\mathrm{n}(\%)$

$10(2 \%)$

Current smoker at time of hospital admission; $\mathrm{n}(\%)$

Body mass index at the time of hospital admission; mean \pm SD

$30 \pm 8$

Outcome

EQ-5D; median (IQR)

$0.77(0.51-0.83)$

ALL, acute lymphocytic leukemia; $A M L$, acute myelogenous leukemia; CNL, chronic myelogenous leukemia.

current smoking, but current smoking performed better in the primary model and was therefore retained in the model. Other patterns of collinearity were not important. As to whether collinearity led to inappropriate exclusion of severity of illness predictors, when the prespecified severity of illness predictors were incorporated into the primary model, they were not significant $(p>0.40)$, and there was no material change in results.

\section{Sensitivity and secondary analyses}

For the sensitivity analysis in which death was assigned a utility score of 0 , a model with three variables available at hospital admission was selected (see online supplementary eTable 3). Older age, higher APACHE III score, and residing at home with informal support (vs residing at home independently) were all associated with lower EQ-5D utility (including death as a utility of 0 ) in this sensitivity analysis.

For the secondary analysis evaluating prediction based on factors available at the time of discharge (uncorrected $\mathrm{R}^{2}=0.18$; see online supplementary eTable 4), results were similar to the primary analysis, with the following variables significantly associated: age, residence at home with informal help, ethnicity, AIDS, current smoking, female sex and BMI. Longer hospital length of stay also was associated with lower utility scores. In the sensitivity analysis of this secondary analysis in which deceased patients were included with a utility score of 0 , only residing at home with informal support (vs residing at home independently) was associated with EQ-5D utility (OR 0.19 $(0.12-0.3), \mathrm{p}<0.001)$.

In the post hoc secondary analysis of the subset of 195 patients with retrospective baseline EQ-5D, the non-cross-validated adjusted $\mathrm{R}^{2}$ in the regression model with versus without baseline EQ-5D utility was 0.32 (vs 0.12 ). As compared with the primary model, BMI, current smoking and baseline independence remained significant or nearly significant, while chronic pulmonary disease became significant (see online supplementary eTable 9).

\section{DISCUSSION}

In this national, multicentre study of ARDS survivors, we explored a large number of possible predictors using modern data science techniques and identified several baseline predictors associated with EQ-5D utility scores at 6-month follow-up. Markers of acute illness severity, however, were not associated with health utility scores. These findings are important considerations for the prediction of QOL after ARDS. Two lifestyle factors, tobacco smoking and obesity appear to be important to long-term outcomes after ARDS. If persistence of tobacco smoking and obesity after hospitalisation were associated with lower health utilities in future studies, they may represent candidate interventions for future studies evaluating methods to improve long-term QOL among survivors of ARDS. Both lifestyle factors remained important predictors of 6-month health utility on multiple secondary and sensitivity analyses.

Our results help confirm prior observations while also identifying several predictors that have not been previously reported. In particular, our study suggests that the degree of independent living at baseline may be an important and easily measured surrogate for baseline impairment and QOL. Other studies have suggested that comorbidities, ${ }^{22} 23$ baseline QOL ${ }^{23} 24$ or severity of trauma $2^{25} 26$ are associated with worse EQ-5D utility scores at follow-up. In our study, comorbidities other than AIDS (as assessed using the APACHE III comorbidity score elements) were not associated with 6-month health utilities among survivors.

Acute illness severity only appeared significant in the sensitivity analysis of our primary model in which mortality was included, demonstrating its relevance to predicting short-term mortality rather than QOL in survivors. In fact, in the sensitivity 
Table 2 Primary regression results

\begin{tabular}{|c|c|c|c|}
\hline Variable & LASSO coefficient* & OLS coefficient* $(95 \% \mathrm{CI})$ & OLS $p$ value \\
\hline \multicolumn{4}{|l|}{ Baseline characteristics } \\
\hline Age & -0.004 & $-0.008(-0.003$ to -0.013$)$ & 0.004 \\
\hline Female sex & -0.189 & $-0.298(-0.148$ to -0.448$)$ & $<0.001$ \\
\hline Hispanic/Latino ethnicity & -0.058 & $-0.334(-0.070$ to -0.598$)$ & 0.013 \\
\hline Resides at home with informal helpt & -0.240 & $-0.576(-0.240$ to -0.911$)$ & $<0.001$ \\
\hline Requires professional help at home OR residence in healthcare facility† & -0.219 & $-0.516(-0.062$ to -0.969$)$ & 0.026 \\
\hline AIDS comorbidity & 0.346 & $0.778(0.160$ to 1.396$)$ & 0.014 \\
\hline Pulmonary comorbidity & -0.001 & $-0.144(-0.382$ to 0.095$)$ & 0.24 \\
\hline Nadir respiratory rate & 0.003 & 0.012 (0 to 0.024$)$ & 0.06 \\
\hline \multicolumn{4}{|l|}{ Lifestyle factors } \\
\hline Current smoker status (at baseline) & -0.179 & $-0.349(-0.195$ to -0.503$)$ & $<0.001$ \\
\hline Body mass index (at baseline) & -0.011 & $-0.019(-0.010$ to -0.028$)$ & $<0.001$ \\
\hline
\end{tabular}

analysis of our secondary model based on patients alive at the time of discharge and incorporating patients who died after hospital discharge with a health utility of 0 , only the degree of independent living at baseline remained in the model, suggesting that acute illness severity was relevant to predicting hospital mortality rather than postdischarge mortality. Our secondary analysis evaluating for collinearity among predictor variables confirmed the lack of association between acute illness severity variables and EQ-5D health utility. Only rarely have prior studies suggested that severity of acute illness is associated with subsequent QOL. ${ }^{27} 28$ The findings in our large cohort offer no support for an association between acute illness severity and postdischarge QOL outcomes. Additionally, no variables associated with ICU treatments, such as mechanical ventilation (eg, plateau or driving pressures, tidal volume), vasopressors or corticosteroids, were associated with health utilities in our cohort.

Tobacco smoking is associated with susceptibility to, and (in some studies) higher mortality from, ARDS. ${ }^{29}$ The present study extends prior observations by documenting an association with lower 6-month health utilities among survivors. This association is likely driven by pre-existing damage from tobacco smoking. However, if ongoing smoking worsens QOL (particularly in patients with new pulmonary dysfunction related to their ARDS), it is possible that targeted smoking cessation interventions among ICU patients could improve long-term outcomes. In another patient population-individuals admitted for an acute myocardial infarction-posthospitalisation decreases in smoking were associated with better patient outcomes at 12 months, suggesting that benefit may still accrue even when tobacco smoking ceases during hospitalisation. ${ }^{31}$ An ICU admission may similarly represent a sustained period of time without access to tobacco, in which smoking cessation interventions may be efficacious. ${ }^{32}$ Further evaluation of the association between persistent smoking and outcomes among ARDS survivors is indicated to explore our hypothesis-generating findings.

While patients with extreme obesity were excluded from ALTOS, patients with higher baseline BMI in our cohort had lower 6-month utility scores after ARDS. The harms associated with obesity at baseline may already be done by the time of ARDS admission. Intentional weight loss after ICU discharge is likely to be complex: on average, ARDS survivors lose almost $20 \%$ of body mass during their acute illness, regaining most of that mass by 1 year. ${ }^{33}$ In older patients, higher BMI at the time of hospital admission is associated with better functional outcomes after an ICU admission. ${ }^{34}$ We suspect that this is a phenomenon specific to low muscle mass among vulnerable elders, whereas the ALTOS survivor population was generally a younger population (median (IQR) of age $=50(40,58)$ years). In general, reduction in BMI has been associated with improvement in QOL, but this is not well studied among ICU survivors. ${ }^{35}$ Further work to clarify the relationship between BMI, QOL and intentional weight loss among ARDS survivors who are obese at the time of hospital admission may be indicated.

Even though patients with end-stage AIDS were excluded from enrolment into our cohort, the 24 patients with AIDS had high mortality (58\%) in our cohort, but the 10 survivors had relatively high health utilities. Recent studies suggest that shortterm survival for patients with AIDS admitted to the ICU are continually improving. ${ }^{36} 37$ While few, if any, studies have addressed long-term QOL outcomes after ARDS for patients with AIDS, our data suggest the possibility that postdischarge QOL for survivors may be favourable. ${ }^{38}$

Race and ethnicity bear a complex relationship to critical illness outcomes. ${ }^{39}{ }^{40}$ Latino ethnicity varied by hospital site in our study. Although hospital site, per se, was not important to the prediction model, it is possible that Latino ethnicity is a surrogate for differences in quality at the hospital level. The large number of centres relative to the number of outcomes makes it difficult to know whether Latino ethnicity is a marker for socioeconomic status or represents variations in care related to hospital site. Since homelessness and non-English speaking status were exclusion criteria from ALTOS, it is unlikely that the association of Latino ethnicity with these socioeconomic factors accounts for our finding. It also means that our findings may not generalise to homeless or non-English-speaking individuals.

The competing risk of death is an important consideration in all studies of outcomes after ARDS or other critical illnesses. ${ }^{41}$ The degree of independent living at the time of hospitalisation for ARDS remained a significant predictor of 6-month QOL in the sensitivity analysis that included deceased patients (assigning them a utility score of 0 , thereby including them in the worst ordinal category, as has been done in prior research ${ }^{42} 43$ ), suggesting that this finding may be robust. In that analysis, APACHE III score replaced other predictors, not surprising given the substantial observed mortality. Further research is 
needed to evaluate optimal mechanisms for analysis of nonmortality outcomes in patient populations with high mortality. ${ }^{44}$

As a limitation of this study, we acknowledge that baseline QOL likely strongly affects postadmission QOL; but is a challenge to validly measure in critical care studies. ${ }^{5}$ The observed association between independent living before hospital admission and postadmission QOL supports this concept, as does our post hoc subgroup analysis of patients in whom a retrospective baseline health utility was measured. This analysis suggested that a substantial proportion of the variability in 6-month health utility is associated with variability in the baseline health utility. This association was also present in the secondary analysis that included deceased patients with an imputed QOL score of 0 .

The association of tobacco smoking with 6-month health utility may also be explained, in part, by smokers having lower baseline QOL, although the post hoc subgroup analysis incorporating baseline QOL suggested that the effect of current tobacco smoking ( $\mathrm{p}=0.06$ in that small subgroup) may be independent of baseline QOL. In studies where baseline QOL has been accurately measured (eg, in elective cardiac surgery ${ }^{45}$ ), impairments in baseline QOL are clearly associated with postdischarge impairments. ${ }^{46}$ However, baseline QOL is difficult to measure in patients with ARDS: recall bias and the use of proxy assessments may introduce inaccuracies in measurement of baseline QOL; the findings of our post hoc subgroup analysis are therefore tentative. ${ }^{14}$ Nevertheless, our findings suggest that patients' independent living status before hospital admission may be a useful and feasible proxy for baseline status and should be considered when evaluating patient outcomes after ARDS. Moreover, we also acknowledge that study patients were drawn from US clinical trials with specific eligibility criteria, and hence, our findings may not be generalisable to all patients with ARDS or other critically ill populations. Specifically, many patients with already end-stage disease-excluded from the ARDS Network clinical trials-may develop ARDS. Should any of these individuals with end-stage disease survive the ICU admission, their postdischarge health utilities may differ substantially from those observed in our cohort.

We acknowledge that our prediction model exhibits a low cross-validated $\mathrm{R}^{2}$, suggesting that it explains only a small amount of the variance in 6-month health utility. However, our decision to report cross-validated $\mathrm{R}^{2}$ was conservative relative to most prior investigations in this field, which did not publish $\mathrm{R}^{2}$ values for their models (and none to our knowledge employed cross-validation), making direct comparison difficult. $^{23} 242728$ A study of QOL among patients with trauma with and without traumatic brain injury had a non-crossvalidated $\mathrm{R}^{2}$ of 0.22 ; in that setting the association between the brain injury and QOL is dominant. ${ }^{26}$ Our primary focus in this study was to build a prediction model for long-term health utility. On the basis of the low $\mathrm{R}^{2}$, our model should not be used for specific prognostication. Instead it identifies candidate predictors for further focused investigation. Our findings also suggest that, in general, variables available during the hospitalisation explain relatively little of the variance in 6-month health utility, an important caveat with any attempt to prognosticate QOL outcomes after ARDS. Although we undertook multiple sensitivity analyses to help confirm robustness of our results, these results should be considered hypothesis generating.

In conclusion, in this novel, data science-driven analysis of 144 candidate predictors in a large, multicentre prospective cohort of ARDS survivors, tobacco smoking and obesity at hospital admission were associated with worse 6-month EQ-5D utility score, while acute critical illness and its treatments were not associated with this outcome. Living independently without assistance at the time of hospital admission was associated with better 6-month EQ-5D utility, while older age, female sex and Hispanic/Latino ethnicity were associated with worse health utility. These observations support further investigations to develop tailored interventions for patients with ARDS, especially those who are current smokers or obese at the time of admission.

\section{Author affiliations}

${ }^{1}$ Center for Humanizing Critical Care, Intermountain Healthcare, Murray, Utah, USA ${ }^{2}$ Department of Medicine, Pulmonary and Critical Care Division, Intermountain Medical Center, Murray, Utah, USA

${ }^{3}$ Pulmonary and Critical Care, University of Utah School of Medicine, Salt Lake City, Utah, USA

${ }^{4}$ Division of Epidemiology, Study Design and Biostatistics Center, University of Utah School of Medicine, Salt Lake City, Utah, USA

${ }^{5}$ Outcomes After Critical Illness and Surgery Group, Johns Hopkins University, Baltimore, Maryland, USA

${ }^{6}$ Division of Pulmonary and Critical Care Medicine, School of Medicine, Johns Hopkins University, Baltimore, Maryland, USA

${ }^{7}$ Department of Psychology, Neuroscience Center, Brigham Young University, Provo, Utah, USA

${ }^{8}$ Department of Physical Medicine and Rehabilitation, School of Medicine, Johns Hopkins University, Baltimore, Maryland, USA

\section{Twitter Follow Samuel Brown at @DrSamuelBrown}

Contributors SMB, ROH, APP, TG and DMN designed the study. SMB, CZ, APP, TG, VDD, DMN and EW analysed and interpreted data. SMB, ROH and DMN drafted the report and all other authors revised it. All authors gave final approval of the report to be published.

Funding National Heart, Lung and Blood Institute (R21HL123433, N01HR56170, R01HL091760, 3R01HL091760-02S1 and contracts HHSN268200536165C to HHSN268200536175C and HHSN268200536179C).

Competing interests None declared.

Ethics approval Intermountain Healthcare IRB.

Provenance and peer review Not commissioned; externally peer reviewed.

\section{REFERENCES}

1 Zambon M, Vincent JL. Mortality rates for patients with acute lung injury/ARDS have decreased over time. Chest 2008;133:1120-7.

2 Needham DM, Davidson J, Cohen $\mathrm{H}$, et al. Improving long-term outcomes after discharge from intensive care unit: report from a stakeholders' conference. Crit Care Med 2012;40:502-9.

3 Puthucheary ZA, Denehy L. Exercise interventions in critical illness survivors: understanding inclusion and stratification criteria. Am J Respir Crit Care Med 2015;191:1464-7.

4 Dowdy DW, Eid MP, Dennison CR, et al. Quality of life after acute respiratory distress syndrome: a meta-analysis. Intensive Care Med 2006;32:1115-24.

5 Oeyen SG, Vandijck DM, Benoit DD, et al. Quality of life after intensive care: a systematic review of the literature. Crit Care Med 2010;38:2386-400.

6 Dowdy DW, Eid MP, Sedrakyan A, et al. Quality of life in adult survivors of critical illness: a systematic review of the literature. Intensive Care Med 2005;31:611-20.

7 Needham DM, Dinglas VD, Bienvenu OJ, et al. One year outcomes in patients with acute lung injury randomised to initial trophic or full enteral feeding: prospective follow-up of EDEN randomised trial. BMJ 2013;346:f1532.

8 The EuroQol Group. EuroQol-a new facility for the measurement of health-related quality of life. Health Policy 1990;16:199-208.

9 Turnbull AE, Rabiee A, Davis WE, et al. Outcome measurement in ICU survivorship research from 1970 to 2013: a scoping review of 425 publications. Crit Care Med 2016;44:1267-77.

10 Angus DC, Carlet J, Brussels Roundtable P. Surviving intensive care: a report from the 2002 Brussels Roundtable. Intensive Care Med 2003;29:368-77.

11 Shaw JW, Johnson JA, Coons SJ. US valuation of the EQ-5D health states: development and testing of the D1 valuation model. Med Care 2005;43:203-20.

12 Walters SJ, Brazier JE. Comparison of the minimally important difference for two health state utility measures: EQ-5D and SF-6D. Qual Life Res 2005;14:1523-32.

13 Pickard AS, Neary MP, Cella D. Estimation of minimally important differences in EQ-5D utility and VAS scores in cancer. Health Qual Life Outcomes 2007;5:70.

14 Dinglas VD, Gifford JM, Husain N, et al. Quality of life before intensive care using EQ-5D: patient versus proxy responses. Crit Care Med 2013;41:9-14. 
15 Reisinger MW, Moss M, Clark BJ, et al. Brief versus full alcohol use disorders identification test in national heart, lung, and blood institute acute respiratory distress syndrome network clinical trials. Crit Care Med 2015;43:e382-5.

16 Naglie G, Krahn MD, Naimark D, et al. Primer on medical decision analysis: Part 3-Estimating probabilities and utilities. Med Decis Making 1997;17:136-41.

17 Tibshirani R, Bien J, Friedman J, et al. Strong rules for discarding predictors in lasso-type problems. J R Stat Soc Series B Stat Methodo/2012;74:245-66.

18 Zhang P. Model selection via multifold cross validation. Ann Stat 1993;21:299-313.

19 Nieuwkerk PT, Tollenaar MS, Oort FJ, et al. Are retrospective measures of change in quality of life more valid than prospective measures? Med Care 2007;45:199-205.

20 van der Heijden GJ, Donders AR, Stijnen T, et al. Imputation of missing values is superior to complete case analysis and the missing-indicator method in multivariable diagnostic research: a clinical example. J Clin Epidemiol 2006;59:1102-9.

21 Peña EA, Slate EH. Global validation of linear model assumptions. J Am Stat Assoc 2006;101:341.

22 Oeyen SG, Benoit DD, Annemans L, et al. Long-term outcomes and quality of life in critically ill patients with hematological or solid malignancies: a single center study. Intensive Care Med 2013;39:889-98.

23 Nisula S, Vaara ST, Kaukonen KM, et al. Six-month survival and quality of life of intensive care patients with acute kidney injury. Crit Care 2013;17:R250.

24 Hofhuis JG, Spronk PE, van Stel HF, et al. Quality of life before intensive care unit admission is a predictor of survival. Crit Care 2007;11:R78.

25 Christensen MC, Banner $C$, Lefering $R$, et al. Quality of life after severe trauma: results from the global trauma trial with recombinant Factor VII. J Trauma 2011;70:1524-31.

26 Gross T, Schüepp M, Attenberger C, et al. Outcome in polytraumatized patients with and without brain injury. Acta Anaesthesiol Scand 2012;56:1163-74.

27 Capuzzo M, Moreno RP, Jordan B, et al. Predictors of early recovery of health status after intensive care. Intensive Care Med 2006;32:1832-8.

28 Orwelius L, Lobo C, Teixeira Pinto A, et al. Sepsis patients do not differ in health-related quality of life compared with other ICU patients. Acta Anaesthesiol Scand 2013;57:1201-5

29 Hsieh SJ, Zhuo H, Benowitz NL, et al. Prevalence and impact of active and passive cigarette smoking in acute respiratory distress syndrome. Crit Care Med 2014;42:2058-68.

30 Calfee CS, Matthay MA, Kangelaris KN, et al. Cigarette smoke exposure and the acute respiratory distress syndrome. Crit Care Med 2015;43:1790-7.

31 Van Spall HG, Chong A, Tu JV. Inpatient smoking-cessation counseling and all-cause mortality in patients with acute myocardial infarction. Am Heart J 2007:154:213-20.
32 Jones $C$, Griffiths RD, Skirrow P, et al. Smoking cessation through comprehensive critical care. Intensive Care Med 2001;27:1547-9.

33 Herridge MS, Cheung AM, Tansey CM, et al. One-year outcomes in survivors of the acute respiratory distress syndrome. N Engl J Med 2003;348:683-93.

34 Ferrante LE, Pisani MA, Murphy TE, et al. Factors associated with functional recovery among older ICU survivors. Am J Respir Crit Care Med Published Online First: 3 Feb 2016. doi:10.1164/rccm.201506-12560C

35 Kroes M, Osei-Assibey G, Baker-Searle R, et al. Impact of weight change on quality of life in adults with overweight/obesity in the United States: a systematic review. Curr Med Res Opin 2016;32:485-508.

36 Barbier F, Roux A, Canet E, et al. Temporal trends in critical events complicating HIV infection: 1999-2010 multicentre cohort study in France. Intensive Care Med 2014;40:1906-15.

37 Mendez-Tellez PA, Damluji A, Ammerman D, et al. Human immunodeficiency virus infection and hospital mortality in acute lung injury patients. Crit Care Med 2010;38:1530-5.

38 Sarkar P, Rasheed HF. Clinical review: respiratory failure in HIV-infected patients-a changing picture. Crit Care 2013;17:228

39 Erickson SE, Vasilevskis EE, Kuzniewicz MW, et al. The effect of race and ethnicity on outcomes among patients in the intensive care unit: a comprehensive study involving socioeconomic status and resuscitation preferences. Crit Care Med 2011;39:429-35.

40 Soto GJ, Martin GS, Gong MN. Healthcare disparities in critical illness. Crit Care Med 2013:41:2784-93.

41 Wolkewitz $\mathrm{M}$, Sommer $\mathrm{H}$. Evaluating mortality in an intensive care unit requires extended survival models. Crit Care 2014;18:415.

42 Lachin JM. Worst-rank score analysis with informatively missing observations in clinical trials. Control Clin Trials 1999:20:408-22.

43 Chen YH, Gould AL, Nessly ML. Treatment comparisons for a partially categorical outcome applied to a biomarker with assay limit. Stat Med 2005;24:211-28.

44 Jones M, Mishra GD, Dobson A. Analytical results in longitudinal studies depended on target of inference and assumed mechanism of attrition. J Clin Epidemiol 2015:68:1165-75.

45 Elliott D, Lazarus R, Leeder SR. Health outcomes of patients undergoing cardiac surgery: repeated measures using Short Form-36 and 15 Dimensions of Quality of Life questionnaire. Heart Lung 2006;35:245-51.

46 Feemster LC, Cooke CR, Rubenfeld GD, et al. The influence of hospitalization or intensive care unit admission on declines in health-related quality of life. Ann Am Thorac Soc 2015; 12:35-45. 